\title{
Low-energy effects of Charged Higgs with general Yukawa couplings
}

\author{
J.L. Diaz Cruz ${ }^{a, b}$, J.J. Godina Nava ${ }^{a}$, G. Lopez Castro ${ }^{a}$ \\ a) Depto. de Fisica, CINVESTAV-IPN, Ap. Postal 14-740, 07000 México D.F. \\ b) Instituto de Fisica, U.A. de Puebla, Puebla, Pue., México
}

\begin{abstract}
We study a model with two Higgs doublets where FCNC are allowed at treelevel. In this model, the interactions of charged Higgs with fermions $\left(H^{ \pm} f \bar{f}^{\prime}\right)$, include a term that is not proportional to the fermion masses, which we constraint using the following low-energy processes: i) tau decays $\left(\tau \rightarrow \nu_{\tau} e \nu_{e}, \nu_{\tau} \mu \nu_{\mu}, \nu_{\tau} \pi\right)$, ii) leptonic decays of pseudoscalar mesons $\left(\pi, K \rightarrow \ell \nu_{\ell}\right)$ and iii) semileptonic bdecays. With these constraints it is possible to make predictions; we illustrate this by presenting the rates for the (FCNC) decay $c \rightarrow u+\gamma$, the (second class-current) decay $\tau \rightarrow \nu_{\tau}+\eta \pi$, and also the theoretical value of the neutron life-time.
\end{abstract}




\section{Introducción}

The standard model (SM) of electroweak interactions [1] has been quite succesfull in confronting the experiments, although it requires the discovery of the top quark and Higgs boson for its confirmation. The existence of the top is widely accepted, and there are already signs of its presence at FNAL, with a mass of 174 $\mathrm{GeV}[2]$. On the other hand, LEP has established a lower bound on the Higgs boson mass of $63.5 \mathrm{GeV}[3]$.

Because of several puzzles that the SM can not explain, e.g. the number of parameters, the fermion family and mass patterns, the hierarchy and naturalness problems etc., many alternatives or extensions to the SM have been proposed. In some of these extensions there are new sources of CP-violation, besides the SM one (which comes from the KM mixing matrix [4]), for instance spontaneous CP-violation [5]. Some extensions contain also flavour changing neutral currents (FCNC) at tree-level, which are absent in the SM [6].

In fact, $\mathrm{CP}$-violation and $\mathrm{FCNC}$ decays have been observed for the downtype quark sector only [7]. For up-type quarks, the SM predicts that $\mathrm{CP}$ and FCNC phenomena will occur at very small rates; for instance, the SM prediction for the $\mathrm{CP}$ asymmetry in top decays is very small, about $10^{-11}$ for the decay $t \rightarrow b \bar{b} c$ [8]. Similarly, the SM result for the FCNC decay $t \rightarrow c+\gamma$ gives a very small branching ratio (BR), about $10^{-12}[9]$, which will not be detectable at future experiments. However, in extensions of the SM with an enlarged Higgs sector, the BR for $t \rightarrow c+\gamma$ can reach a value of $10^{-6}$, and the CP asymmetry for the decay $t \rightarrow b+\tau^{+} \nu$ can be about 6 orders of magnitude larger than the SM value, namely $O\left(10^{-5}\right)[10]$, which could be tested at future colliders. 
In models with two Higgs doublets $\left(\Phi_{1}\right.$ and $\left.\Phi_{2}\right)$ [11], it was usual to avoid FCNC mediated by neutral Higgs bosons, by imposing a discrete symmetry $\Phi_{1} \rightarrow$ $\Phi_{1}, \quad \Phi_{2} \rightarrow-\Phi_{2}$, which could be broken only softly (i.e. by terms of dimension two). The absence of FCNC was imposed in these models because of the belief that in order to satisfy the current experimental limits, the Higgs bosons have to be very heavy, which in turn would lead to violations of unitarity [12]. However, as it was noticed in [13], FCNC can also be suppressed with relatively light Higgs masses $\left(m_{h}<O(1 \mathrm{TeV})\right)$, provided that the couplings $h q_{i} \bar{q}_{j}$ behaves like $\left(m_{i} m_{j}\right)^{1 / 2}$ instead of $m_{i}^{2}$, as it was assumed before.

In this paper we are interested in evaluating the constraints that can be imposed on the parameters of a two-Higgs doublet model that allows FCNC at tree-level, particularly in the charged Higgs parameters, using present low-energy data. Once these constraints are known, one can use them to make quantitative predictions for new phenomena, which will be the signature of the new physics incorporated in the model [14].

The neutral Higgs sector of two-Higgs doublet models with FCNC, has been already studied in Refs. [13,15], focusing on both FCNC and CP phenomena. It has been also shown in [15], that the type of Yukawa couplings needed to supress FCNC, can be obtained in models where the fermion masses are derived using some ansatz for the mass matrices, e.g. the Fritzch type.

We shall follow a similar approach as in $[13,16]$, where the focus is in constraining the couplings that would signal the new physics, rather than obtaining limits only on the Higgs masses, as has been usual in most studies. We follow this approach because our motivation is to use the properties of the charged Higgs, as a tool to test the models that attempt to explain the fermionic mass pattern. 
The organization of this paper is as follows. In section 2 we explain some details of the model. Section 3 contains our main results, namely the analysis of several low-energy processes used to constraint the interactions of the charged Higgs with fermions. The data used in the work has been selected mostly to make a first evaluation of the relevant parameters of the model, rather than to do an extensive analysis of the parameter space. These constraints are used in section 4 to predict the rates for: the FCNC decay $c \rightarrow u+\gamma$, and the (second class current) decay $\tau^{-} \rightarrow \nu_{\tau}+\eta+\pi^{-}$. We consider also the apparent discrepancy between the SM prediction and the measured neutron life-time, to see if it could be resolved within our model. We have choosen these processes because they are sensitive to the appearence of a charged scalar, although there may be other ones where the predictions could be even more sensitive. Finally, our conclusions are presented in sect. 6 .

\section{The Model}

The model includes two Higgs doublets, $\Phi_{1}$ and $\Phi_{2}$, of equal hypercharges $(\mathrm{Y}=1)$, such that after spontaneous symmetry breaking it contains five Higgs bosons. The most general Yukawa interaction lagrangian can be written as follows ${ }^{\# 1}$ :

$$
L_{Y}=\bar{\Psi}_{L i}^{0}\left(F_{i j} \tilde{\Phi}_{1}+\xi F_{i j}^{\prime} \tilde{\Phi}_{2}\right) U_{R j}^{0}+\bar{\Psi}_{L i}^{0}\left(G_{i j} \Phi_{2}+\xi G_{i j}^{\prime} \Phi_{1}\right) D_{R j}^{0}+\text { h.c. }
$$

where $\Psi_{L i}^{0}=\left(U_{i}^{0}, D_{i}^{0}\right)_{L}$ denote the $S U(2)$ quark doublet, and $U_{R j}^{0}, D_{R j}^{0}$ are the quark singlets (the superscript 0 is used for weak eigenstates, whereas $i, j$ denote

\#1 A similar piece of the lagrangian should be understood for the Yukawa couplings of the leptons. 
generation number). $F, F^{\prime}, G$ and $G^{\prime}$ are dimensionless $3 \times 3$ matrices characterizing the Yukawa couplings and $\xi$ parametrizes the discrete symmetry breaking.

A $U(1)_{e m}$-invariant VEV is given by $<\Phi_{1}>^{T}=\left(0, \frac{v_{1}}{\sqrt{2}}\right),<\Phi_{2}>^{T}=\left(0, \frac{v_{2} e^{i \delta}}{\sqrt{2}}\right)$ where the phase $\delta$ signals the violation of CP in the Higgs sector. The scalar spectrum consists of a charged Higgs pair $\left(H^{ \pm}\right)$and three neutral Higgs bosons $\left(A^{0}, h^{0}, H^{0}\right)$. After SSB, we obtain from Eq.(2.1) the following expressions for the fermion mass matrices,

$$
\begin{aligned}
& M_{u}^{0}=\left(F+e^{-i \delta} \xi \frac{v_{2}}{v_{1}} F^{\prime}\right) \frac{v_{1}}{\sqrt{2}} \\
& M_{d}^{0}=\left(G+e^{-i \delta} \xi \frac{v_{1}}{v_{2}} G^{\prime}\right) \frac{v_{2}}{\sqrt{2}} .
\end{aligned}
$$

In order to diagonalize these mass matrices we shall work in a basis where $M_{u}^{0}$ is diagonal $\left(M_{u}^{0}=M_{u}\right)$. Then the mass matrix for down-type quarks can be diagonalized through the following transformations,

$$
M_{d}=V_{L}^{+} M_{d}^{0} V_{R}=\frac{v_{2}}{\sqrt{2}} V_{L}^{+}\left[G+e^{-i \delta} \xi \frac{v_{1}}{v_{2}} G^{\prime}\right] V_{R}
$$

$V_{L}$ can be identified as the KM mixing matrix. Then, the interaction between the quarks (mass eigenstates) and the charged Higgs boson is given by the following expresion,

$$
\begin{aligned}
L_{q_{i} \bar{q}_{j} H^{ \pm}}= & \frac{g}{\sqrt{2} m_{W}} H^{+} \bar{U}\left[\cot \beta V_{L}^{+} M_{d} R+\tan \beta V_{L} M_{u} L+\right. \\
& \left.\xi e^{-i \delta_{1}} M_{1} \Gamma L+\xi e^{-i \delta_{2}} M_{2} \Gamma^{\prime} R\right] D+\text { h.c. }
\end{aligned}
$$

where $\Gamma=F^{\prime} V_{L}, \Gamma^{\prime}=G^{\prime} V_{L}$ and $(\mathrm{L}, \mathrm{R})$ denote the helicity projection operators. 
The phases $\delta_{1}, \delta_{2}$ are given by:

$$
\begin{aligned}
& \tan \delta_{1}=-\frac{\sin \delta}{(\cos \delta+\cot \beta)}, \\
& \tan \delta_{2}=-\frac{\sin \delta}{(\cos \delta+\tan \beta)} .
\end{aligned}
$$

The parameters $M_{1,2}$ are given by: $M_{1}=\sqrt{2} m_{W} s_{1} / g$ and $M_{2}=\sqrt{2} m_{W} \cot \beta s_{1} / g$, with $\tan \beta \equiv v_{2} / v_{1}, s_{1}=\left[\sec ^{2} \beta-4 \sin ^{2} \beta \sin ^{2} \delta / 2\right]^{1 / 2}$, and $g$ denotes the $\operatorname{SU}(2)$ coupling constant.

A similar expression is obtained for leptons. In this paper we assume that neutrinos are massless, then one can choose a basis where the coupling between the $W^{ \pm}$and leptons is diagonal. On the other hand, because of the terms that

produce FCNC, the couplings $H^{+} \ell \nu_{\ell}$ are not diagonal in general. However, in the present work we shall neglect the effects of the non-diagonal terms in the $H^{+} \ell_{i} \nu_{j}$ vertex.

\section{Bounds on the Yukawa couplings of the charged Higgs}

In this section we shall study the limits that can be imposed on the charged Higgs-fermions couplings from low-energy data. The focus will be on the quantities that will be used later for the predictions of the model. We shall attempt to obtain limits only for some sets of typical values of parameters, rather than doing an extensive analysis of the complete regions of parameter space. We believe that this will illustrate the most important features of our model.

In the following analysis, we shall neglect in the vertices $H^{ \pm} f_{i} \overline{f_{j}}$ the contributions proportional to the masses of s- and b-quarks, with respect to c- and t- masses. 
From an analysis of Yukawa interactions using some ansatz for the mass matrices [15], it was found that $\Gamma^{\prime}$ (in Eq.(2.5)) is neglibible with respect to $\Gamma$, because of the hierarchy of quark masses and mixing angles. Thus, with this approximation the vertex $H^{ \pm} f_{i} \overline{f_{j}}$ for light fermions reduces to the form:

$$
V_{H^{ \pm} f_{i} \bar{f}_{j}}=\frac{g m_{H}}{2 m_{W}} e^{i \delta_{1}} \lambda_{i j}\left(1-\gamma_{5}\right)
$$

where $\lambda_{i j}=\xi M_{1} \Gamma_{i j} / m_{H}$. The dimensionless factors $\lambda_{i j}$ appear in a natural way in our calculations of the following sections, because we are considering weak decays of light particles $\left(m_{f}<<m_{H^{ \pm}}\right)$and then our effective lagrangian corresponds to a four-fermion local interaction. Our results are expressed as bounds for $\lambda_{i j}$ which are summarized in table 1.

\subsection{CONSTRAints FROM LEPTONIC TAU DECAYS}

The recent experimental results on the mass and leptonic branching ratios of the tau have reached such precision [17], that they allow now to derive bounds on new physics. These measurements will be used to obtain bounds on the parameters $\lambda_{e \nu}, \lambda_{\mu \nu}, \lambda_{\tau \nu}$ defined in Eq.(3.1).

The width for the decay $\tau \rightarrow \nu_{\tau}+l+\nu_{l}$, including $W^{ \pm}$and $H^{ \pm}$contributions can be written as follows,

$$
\Gamma=\frac{G_{F}^{2} m_{\tau}^{5}}{192 \pi^{3}} f\left(z_{l}\right)\left(1+h_{R C}\right)\left[1+\frac{\lambda_{\tau \nu_{\tau}}^{2} \lambda_{\ell \nu_{\ell}}^{2}}{4}\right]
$$

where $z_{l}=\frac{m_{l}^{2}}{m_{\tau}^{2}}, f(z)=1-8 z+8 z^{3}-z^{4}-12 z^{2} \log z$. The effect of radiative corrections $^{\# 2}$ is included in the function $h_{R C}$, which has been evaluated in [18].

\#2 In the calculations of the present and forthcoming sections for SM allowed processes, we shall use the approximation that radiative corrections are the same for the amplitudes mediated by $W^{ \pm}$and $H^{ \pm}$, since this accounts to neglect small terms of order $O\left(\alpha \lambda_{i j} \lambda_{l \nu}\right)$. 
The previous decays are clean predictions of the SM if one uses the value of $G_{F}=1.16637(2) \times 10^{-5} \mathrm{GeV}^{-2}$, as obtained from muon decay. In order to bound the charged Higgs contribution, we shall require it to lay below the experimental uncertainty. $^{\# 3}$ Using the data: $B R\left(\tau \rightarrow e \nu_{e} \nu_{\tau}\right)=0.1789 \pm 0.0014, B R(\tau \rightarrow$ $\left.\mu \nu_{\mu} \nu_{\tau}\right)=0.1734 \pm 0.0016$ [17], we obtain the bounds that appear in the first two entries of table 1, namely:

$$
\begin{gathered}
\lambda_{\tau \nu_{\tau}} \lambda_{e \nu_{e}}<0.22 \\
\lambda_{\tau \nu_{\tau}} \lambda_{\mu \nu_{\mu}}<0.23
\end{gathered}
$$

For completeness, the bound obtained from muon decay is also given in table $1^{\# 4}$.

As it will be explained in the following sections, one does not have to know the bounds separately for each of the $\lambda_{i j}$ 's since only the previous combination will appear in the quantities of interest in the present paper.

\subsection{Constraints from Leptonic DeCays of PSEUdoscalar MESOnS}

The leptonic decays of charged pseudoscalar mesons $P^{ \pm} \rightarrow l^{ \pm}+\nu_{l},(P=\pi, K)$, can be used to obtain bounds for the products: $\lambda_{u d} \lambda_{l \nu_{l}}, \lambda_{u s} \lambda_{l \nu_{l}}$. According to [19], the SM result for the decay width of the meson P, can be written as follows:

$$
\Gamma_{S M}=\frac{G_{F}^{2}\left|V_{u j}\right|^{2} f_{P}^{2} m_{P} m_{l}^{2}}{8 \pi}\left[1-\frac{m_{l}^{2}}{m_{P}^{2}}\right]^{2}\left(1+h_{R C}\right),
$$

where $f_{P}$ is the decay constant of the pseudoscalar meson $P$, which is defined from: $<0\left|\bar{q}_{j}(x) \gamma_{\mu} \gamma_{5} u\right| P>=i f_{P} p_{\mu} e^{-i p \cdot x}\left(q_{j}=d, s\right.$ for $\pi^{+}$and $K^{+}$mesons, respectively); the function $h_{R C}$ includes the effect of radiative corrections [18].

\#3 This procedure will be used also in the forthcoming sections, unless otherwise specified.

\#4 If one considers non-diagonal terms in the vertex $H^{+} \ell_{i} \nu_{j}$, then the previous bound would translate into a bound for $\sum_{i, j} \lambda_{\tau \nu_{i}} \lambda_{\ell \nu_{j}}$. 
The tree-level contribution of the charged Higgs to this decay width is obtained by adding the graph where the $\mathrm{W}$ boson is substituted by the charged Higgs. Because of parity-invariance, only the pseudoscalar part of the Higgs-fermion interaction contributes to the amplitude, which is then given by:

$$
M=\frac{G_{F}}{\sqrt{2}}\left[V_{j u}<0\left|\bar{q}_{j}(x) \gamma_{\mu} \gamma_{5} u\right| P>l_{\mu}+\lambda_{j u} \lambda_{\ell \nu}<0\left|\bar{q}_{j}(x) \gamma_{5} u\right| P>l_{S}\right]
$$

where the leptonic currents $l_{\mu}$ and $l_{S}$ are given by: $l_{\mu}=\bar{l} \gamma_{\mu}\left(1-\gamma_{5}\right) \nu_{l}$, $l_{S}=\bar{l}\left(1-\gamma_{5}\right) \nu_{l}$, respectively.

The hadronic matrix element of the pseudoscalar current can be obtained from the axial one by using the relation:

$$
<0\left|\bar{q}_{j} \gamma_{5} u\right| P>\equiv \frac{i f_{P} m_{P}^{2}}{\left(m_{j}+m_{u}\right)}=A_{1} V_{j u}
$$

where $A_{1}=\frac{2 m_{P}^{2}}{V_{j u} m_{\ell}\left(m_{j}+m_{u}\right)}$.

Then, the total width is given by,

$$
\Gamma=\Gamma_{S M}\left(1+2 A_{1} \lambda_{j u} \lambda_{\ell \nu}+A_{1}^{2} \lambda_{j u}^{2} \lambda_{\ell \nu}^{2}\right)
$$

It has become convenient in tests of $e-\mu$ universality, to evaluate the ratio:

$$
R_{e / \mu}(P)=\frac{\Gamma\left(P \rightarrow e \nu_{e}(\gamma)\right)}{\Gamma\left(P \rightarrow \mu \nu_{\mu}(\gamma)\right)}
$$

which is independent of $f_{P}$. By using the extreme values allowed by one standard deviation in the ratios: 
$R_{e / \mu}^{e x p}(\pi) / R_{e / \mu}^{S M}(\pi)=0.9966 \pm 0.0031[19]$, and $R_{e / \mu}^{e x p}(K) / R_{e / \mu}^{S M}(K)=0.965 \pm 0.043$

[7], we obtain the following bounds:

$$
\begin{gathered}
\left|\lambda_{u d}\left(\lambda_{e \nu}-\frac{m_{e}}{m_{\mu}} \lambda_{\mu \nu}\right)\right|<6.44 \times 10^{-7}, \\
\left|\lambda_{u s}\left(\lambda_{e \nu}-\frac{m_{e}}{m_{\mu}} \lambda_{\mu \nu}\right)\right|<1.84 \times 10^{-6} .
\end{gathered}
$$

\subsection{Constraints From the DeCAy $\tau \rightarrow \nu_{\tau}+\pi$}

We will use this decay to get a bound on the product $\lambda_{u d} \lambda_{\tau \nu}$. The contribution from the charged Higgs to the decay amplitude can be included along similar lines of the previous sections. In order to use the radiative corrections from Ref. [20,18], we will work out the following ratio:

$$
\frac{\Gamma\left(\tau \rightarrow \nu_{\tau}+\pi\right)}{\Gamma\left(\tau \rightarrow e \nu_{e} \nu_{\tau}\right)}=\frac{\Gamma^{S M}\left(\tau \rightarrow \nu_{\tau}+\pi\right)\left[1+\delta_{\tau \pi}\right]}{\Gamma^{S M}\left(\tau \rightarrow e \nu_{e} \nu_{\tau}\right)\left[1+\delta_{\tau e}\right]}
$$

where the SM contribution has been factored out, and the terms that include the charged Higgs contributions are given by:

$$
\delta_{\tau \pi}=\frac{4 m_{\pi}^{2} \lambda_{u d} \lambda_{\tau \nu}}{m_{\tau} V_{u d}\left(m_{d}+m_{u}\right)}
$$

and $\delta_{\tau e}=\lambda_{\tau \nu}^{2} \lambda_{e \nu}^{2} / 4$, which has been bounded in section 3.1 .

In order to bound $\delta_{\tau \pi}$, we proceed as follows: for the left-hand side of Eq.(3.9) we use the experimental data $[7,17]$,

$$
\frac{\Gamma^{\exp }\left(\tau \rightarrow \nu_{\tau}+\pi\right)}{\Gamma^{\exp }\left(\tau \rightarrow e \nu_{e} \nu_{\tau}\right)}=0.648 \pm 0.023
$$

For the SM part we use the result of Ref. [20], which includes $O(\alpha)$ radiative corrections, namely 


$$
\frac{\Gamma^{S M}\left(\tau \rightarrow \nu_{\tau}+\pi\right)}{\Gamma^{S M}\left(\tau \rightarrow e \nu_{e} \nu_{\tau}\right)}=0.619 \pm 0.001
$$

Then including the bound (3.3) for $\delta_{\tau e}$, we find $\delta_{\tau \pi}=0.035 \pm 0.039$, which can be translated into a limit for the product of couplings:

$$
\lambda_{u d} \lambda_{\tau \nu}<2.6 \times 10^{-2}
$$

Let us comment that this particular combination of couplings will appear in the evaluation of the second-class decay of the tau (section 4.2).

\subsection{Constraints From the DeCay $b \rightarrow u+\ell \nu_{\ell}$}

The measurements of the decay rates of heavy quarks can also be used to extract information on the couplings $\lambda_{Q q}$. In particular, there are measurements of the inclusive decay $B \rightarrow X \ell \nu_{\ell}$, which allow to put bounds on the coupling $\lambda_{b u}$.

If we consider only the cases when $l=e, \mu$, then the decay width for the semileptonic b decay including $H^{ \pm}$contributions, is written as follows:

$$
\Gamma\left(b \rightarrow u+\ell+\nu_{\ell}\right)=\Gamma_{0} f\left(z_{l}\right) \eta\left|V_{u b}\right|^{2}\left[1+\frac{\lambda_{\tau \nu}^{2} \lambda_{e \nu}^{2}}{4\left|V_{u b}\right|^{2}}\right]
$$

where $z_{l}=m_{\ell}^{2} / m_{b}^{2}, \Gamma_{0}=G_{F}^{2} m_{b}^{5} / 192 \pi^{3}$, and the phase space factor $f(z)$ is defined in Eq.(3.2).

For light leptons $(\ell=e, \mu)$, one can set $f\left(z_{\ell}\right) \simeq 1$. The factor $\eta$ in Eq.(3.12) includes effects from perturbative QCD corrections, and has been estimated to be $\simeq 0.86[21]$. 
In order to extract the bound on the factor $\lambda_{b u} \lambda_{e \nu}$, we use the theoretical predictions for the ratio $R_{B / b}=\frac{\Gamma\left(B \rightarrow \rho+e \nu_{e}\right)}{\Gamma\left(b \rightarrow u+e \nu_{e}\right)} \simeq 3.5-14 \%$ [22], which can be combined with the measured values of the life-time $\tau_{B}=(1.49 \pm 0.04) \times 10^{-12} \mathrm{sec}$ [23] and $B R\left(B \rightarrow \rho+l \nu_{l}\right)<(1.6-2.7) \times 10^{-4}[24]$, as follows:

$$
\Gamma\left(b \rightarrow u+\ell \nu_{\ell}\right)=\frac{B R\left(B \rightarrow \rho+\ell \nu_{\ell}\right)}{\tau_{B} R_{B / b}} .
$$

Since $\left|V_{u b}\right|$ is poorly known, and in fact is expected to be obtained from the previous decay, we shall neglect the SM contribution in order to estimate a bound on $\lambda_{b u}$. We obtain $\left(\right.$ for $m_{b}=4.8 \mathrm{GeV}$ ):

$$
\lambda_{b u} \lambda_{e \nu}<1.43 \times 10^{-2},
$$

which appears in the summary table 1.

\subsection{Constraints from the Decay $b \rightarrow c+\tau \nu_{\tau}$.}

To extract the parameter $\lambda_{b c}$, which corresponds to the remmaining $H^{ \pm} f_{i} \bar{f}_{j}$ couplings needed to make our predictions, we shall use the decay $b \rightarrow c+\tau \nu_{\tau}$.

The BR for this decay has been meassured recently [25], $\left(B R\left(b \rightarrow c+\tau \nu_{\tau}\right)=\right.$ $\left.\left(4.2_{-0.68}^{+0.72} \pm 0.46\right) \times 10^{-2}\right)$, a result that is in fact significantly above the SM prediction $^{\# 5}(B R \simeq 2.5 \%)$.

The amplitude for the decay width, including $W^{ \pm}$and $H^{ \pm}$contributions, is evaluated along the same lines as before. However, in order to evaluate the 3body phase space for this case, with two masses in the final state that can not be

\#5 The decay width for $\Gamma\left(b \rightarrow c+\ell \nu_{\ell}\right), \ell=e, \mu$, can not be used because its value is used to fix $V_{b c}$. 
neglected, we choose to perform a numerical integration. Thus, the decay width can be written as follows,

$$
\Gamma\left(b \rightarrow c \tau \nu_{\tau}\right)=\Gamma_{S M}\left(1+\frac{\lambda_{b c}^{2} \lambda_{\tau \nu}^{2}}{4\left|V_{b c}\right|^{2}}\right)
$$

where $\Gamma_{S M}$ has the following form,

$$
\Gamma_{S M}=\frac{G_{F}^{2} m_{b}^{5}\left|V_{b c}\right|^{2}}{192 \pi^{3}} f\left(\xi_{\tau}, \xi_{c}\right)\left[1-\frac{2 \alpha_{s}\left(m_{b}\right)}{3 \pi}\left(\pi^{2}-\frac{25}{4}\right)\right]
$$

where

$$
\begin{aligned}
f\left(\xi_{\tau}, \xi_{c}\right) & =12 \int_{2 \sqrt{\xi_{c}}}^{1+\xi_{c}-\xi_{\tau}} d x \int_{y^{-}}^{y^{+}} d y W(x, y) \\
& =12 \int_{2 \sqrt{\xi_{c}}}^{1+\xi_{c}-\xi_{\tau}} d x \int_{y^{-}}^{y^{+}} d y H(x, y)
\end{aligned}
$$

with $\xi_{i}=\frac{m_{i}^{2}}{m_{b}^{2}}, i=\tau, c$. The $W^{ \pm}$and $H^{ \pm}$contributions, $\mathrm{W}(\mathrm{x}, \mathrm{y})$ and $\mathrm{H}(\mathrm{x}, \mathrm{y})$, are:

$$
\begin{aligned}
W(x, y)= & (2-x-y)\left(-1-\xi_{\tau}-\xi_{c}+x+y\right) \\
H(x, y)= & y\left(1+\xi_{\tau}-\xi_{c}-y\right)+x\left(1+\xi_{c}-\xi_{\tau}-x\right) \\
& -(2-x-y)\left(-1-\xi_{\tau}-\xi_{c}+x+y\right)
\end{aligned}
$$

and the integration limits for $y$ are given by

$$
y^{ \pm}=\frac{2 E_{\tau}^{\max , \min }}{m_{b}}
$$

where $E_{\tau}$ is the energy of $\tau$ in the $\mathrm{b}$ rest frame. This result differs from the one presented in Ref. [26], because in our case there is only a new right-handed term, 
then there are no interference terms. The numerical result for the SM contribution to the decay width is $\left(m_{b}=4.8 \mathrm{GeV}, m_{c}=1.5 \mathrm{GeV}\right)$ :

$$
\Gamma_{S M}=\left|V_{c b}\right|^{2} 5.03 \times 10^{-9} \mathrm{MeV},
$$

which gives $B R_{S M} \simeq 2.5 \%$ for $\left|V_{c b}\right|=0.046 \pm 0.005$ [7].

Then, by using the experimental value for $b \rightarrow c \tau \nu$ and the SM result in Eq.(3.14), we obtain the following bound:

$$
\lambda_{b c} \lambda_{\tau \nu}<9.6 \times 10^{-2}
$$

\section{Predictions from the model}

In this section we use the bounds on $\lambda_{i j}$ obtained in the previous section to make some predictions of the model.

\subsection{The DECAY $c \rightarrow u+\gamma$}

One process which could receive an important contribution from the charged Higgs, is the decay $c \rightarrow u+\gamma$. Here we could expect a similar behavior as in top quark [10], where the contribution from the charged and neutral Higgs can give $B R(t \rightarrow c \gamma)<10^{-6}$, which is 6 orders of magnitude larger than the SM prediction. The Feynman graphs are shown in fig. 1. The SM contribution will be omited, since the resulting $\mathrm{BR}$ is negligible $\left(O\left(10^{-15}\right)\right)$ [27]. Using the methods of Ref. 
[28] to evaluate the FCNC decays width of $q_{i} \rightarrow q_{j}+\gamma$ :

$$
\Gamma(c \rightarrow u+\gamma)=\frac{\alpha G_{F}^{2} m_{c}^{5} \lambda_{b c}^{2} \lambda_{b u}^{2}}{128 \pi^{4}}\left[I_{1}-\frac{I_{2}}{3}\right]^{2},
$$

where the functions that result from the evaluation of the loop-integrals are ${ }^{\# 6}$ :

$$
\begin{gathered}
I_{1}=\left(1-6 z+3 z^{2}+2 z^{3}-6 z^{2} \log z\right) /(1-z)^{4}, \\
I_{2}=\left(2+3 z-6 z^{2}+z^{3}+6 z \log z\right) /(1-z)^{4}
\end{gathered}
$$

with $z=\frac{m_{b}^{2}}{m_{H}^{2}}$. We have using the approximation $m_{b}^{2}>>m_{c}^{2}>>m_{u}^{2}$.

The branching ratio can be evaluated as follows,

$$
B R(c \rightarrow u+\gamma)=\frac{\Gamma(c \rightarrow u+\gamma)}{\Gamma_{S M}\left(c \rightarrow s+l \nu_{l}\right)} B R^{\exp }\left(c \rightarrow s+l \nu_{l}\right)
$$

Using the SM result for the semileptonic decay of a heavy quark Eq.(3.15), we obtain:

$$
\frac{\Gamma(c \rightarrow u+\gamma)}{\Gamma_{S M}\left(c \rightarrow s \ell \nu_{\ell}\right)}=\frac{3 \alpha}{2 \pi}\left[I_{1}-I_{2} / 3\right]^{2} \frac{\lambda_{b c}^{2} \lambda_{b u}^{2}}{\left|V_{c s}\right|^{2}}
$$

Then, using the experimental result for the BR of the semileptonic decay, namely $B R(c \rightarrow s+\ell \nu)=(17.2 \pm 1.9) \%$ [7] and taking the bound $\lambda_{b c} \lambda_{b u}<6.24 \times 10^{-3}$ we obtain: $B R(c \rightarrow u+\gamma)<1.8 \times 10^{-11}$, for $\left|V_{c s}\right| \simeq 1$, and $z=1 / 300$, which is well above the SM prediction, but still far from the experimental limits. Thus, it is unlikely that this result can be tested at the proposed tau-charm factory [29], which is expected to produce only $O\left(10^{8}\right)$ c-pairs.

\#6 The form of these functions agree with the ones presented in the literature [28], provided one takes the appropiate limit. 
If the QCD corrections, as estimated in [27], are included, the BR increases one order of magnitud, but this value is still far from the experimental reach ${ }^{\# 7}$.

\subsection{Second Class-CURRENTS in tau DeCAys}

Another application of the bounds obtained before is on the rate for the secondclass decay $\tau \rightarrow \nu_{\tau}+\eta \pi$, which has been bounded experimentaly, namely $B R<$ $3.4 \times 10^{-4}$ [30], which is still above the SM prediction due to isospin breaking [31] $\simeq 10^{-6}$. Charged scalar can mediate this process and give a genuine contribution to second class-current [32].

The contribution of the charged Higgs to this decay could be enhanced by the scalar resonance $a_{0}^{-}(980)$. The corresponding amplitude can be written as follows,

$$
M=\frac{4 G_{F} V_{u d}^{*} \lambda_{u d} \lambda_{\tau \nu}}{\sqrt{2}} \overline{u_{\nu}}\left(1-\gamma_{5}\right) u_{\tau}<\eta \pi|\bar{u} d| 0>.
$$

The hadronic matrix element is written as:

$$
<\eta \pi|u \bar{d}| 0>=\frac{S_{a_{0}} g_{\eta \pi a_{0}}}{m_{a_{0}}^{2}-q^{2}-i \Gamma_{a_{0}} m_{a_{0}}}
$$

where $q=\left(P_{\tau}-p_{\nu}\right)$, and the scalar coupling of the $a_{0}$ is defined as $<a_{0}|\bar{u} d| 0>=S_{a_{0}}$. This constant can be written in terms of the vector coupling of the $a_{0}\left(<a_{0}\left|\bar{u} \gamma_{\mu} d\right| 0>=i f_{a_{0}} p_{\mu} e^{-i p x}\right)$, namely [32]: $S_{a_{0}}=f_{a_{0}} m_{a_{0}}^{2} /\left(m_{d}-m_{u}\right) \simeq$ $(0.55 \mathrm{GeV})^{2}$ where $f_{a_{0}} \simeq 2 \mathrm{MeV}$ [32]. The $a_{0} \eta \pi$ coupling constant is extracted from the total width of the $a_{0}^{-}$, giving $g_{a_{0}^{-} \eta \pi}=(2.02 \pm 0.13) \mathrm{GeV}$.

\#7 The inclusion of the neutral Higgs bosons in the loops could enhance this result, but in that case the analysis depends on more free parameters. 
Substituting the previous relations in the squared amplitude and including the phase-space factor, one finds:

$$
\Gamma\left(\tau \rightarrow \nu_{\tau}+\eta \pi\right)=C_{0} S_{a_{0}}^{2} \lambda_{u d}^{2} \lambda_{\tau \nu_{\tau}}^{2}
$$

where $C_{0}=(1.15 \pm 0.22) \times 10^{-11} \mathrm{GeV}^{-3}$. In order to obtain a numerical result, we shall use the bound obtained for $\lambda_{u d} \lambda_{\tau \nu_{\tau}}$ Eq.(3.11), which was extracted from $\tau \rightarrow \nu_{\tau} \pi$ and leptonic tau decay. Thus we find: $B R(\tau \rightarrow \nu+\eta \pi)<4 \times 10^{-5}$, which is consistent with the present experimental bound [7]. If the $\lambda_{i j}{ }^{6} s$ saturate the bound on their values, the BR would be above the SM prediction, and this could be testable at the proposed tau -charm factories [29].

\subsection{Neutron Beta DeCAy}

Another interesting point to be considered within our model is neutron beta decay. Within the standard model, the neutron lifetime can be predicted [33], in terms of the ratio of axial and vector form factors, $\lambda^{\prime}=g_{A} / f_{V}=-1.2573 \pm 0.0028$ [7], and the quark mixing angle $V_{u d}=0.9744 \pm 0.0010$ [7]. The value obtained for the neutron lifetime, $\tau_{n}=(900 \pm 4) s e c$, is two standard deviations above the measured $^{\# 8}$ value $\tau_{n}=(889.1 \pm 2.1) \sec [7]$. As it will be shown below, the charged Higgs contribution of our model could provide a solution to this discrepancy.

Following reference [34] we write an effective Hamiltonian for neutron beta decay, including $W^{ \pm}$and $H^{ \pm}$contributions, as follows:

$$
\begin{aligned}
H_{e f f}= & \frac{G_{F}}{\sqrt{2}} V_{u d}\left[\bar{u}_{p} \gamma_{\mu}\left(f_{V}+g_{A} \gamma_{5}\right) u_{n} \bar{u}_{e} \gamma_{\mu}\left(1-\gamma_{5}\right) u_{\nu_{e}}\right. \\
& \left.+\frac{\lambda_{u d}}{V_{u d}} \lambda_{e \nu_{e}} \bar{u}_{p}\left(f_{S}+g_{P} \gamma_{5}\right) u_{n} \bar{u}_{e}\left(1-\gamma_{5}\right) u_{\nu_{e}}\right]
\end{aligned},
$$

where the scalar and pseudoscalar form factors, $f_{S}$ and $g_{P}$, arise from the Higgs

\#8 It is important to mention that both values for $\tau_{n}$ agree if $\left|\lambda^{\prime}\right|$ increases up to 1.266 . 
mediated interaction. We can write the form factor $f_{S}$ in terms of charged Higgs parameters as follows:

$$
\lambda^{\prime \prime} \equiv f_{S} / f_{V}=\frac{\lambda_{u d} \lambda_{e \nu}\left(m_{n}-m_{p}\right)}{V_{u d}\left(m_{d}-m_{u}\right)} .
$$

We do not write the corresponding expression for the $g_{P}$ form factor because it gives a negligible contributions to the neutron decay rate.

Thus, the total decay rate can be written as follows:

$$
\begin{aligned}
\tau_{n} & =\frac{1}{\Gamma\left(n \rightarrow p e^{-} \nu_{e}\right)} \\
& =\frac{2 \pi^{3}\left(1-\Delta_{\beta}+\Delta_{\mu}\right)}{G_{F}^{2} m_{e}^{5}\left|V_{u d}\right|^{2}\left(1+3 \lambda^{\prime 2}+\lambda^{\prime \prime 2}\right) f_{1}},
\end{aligned}
$$

where $f_{1}=1.71645 \pm 0.00015$ is the phase space factor including outer [35] e.m. radiative corrections. The inner e.m. radiative corrections are included in $\Delta_{\beta}-$ $\Delta_{\mu} \simeq 2.34 \%$. A comparison of (eq. 4.7) with $\tau_{n}^{\text {exp }}$ gives $f_{S} / f_{V} \simeq 0.2477$ or equivalently, $\lambda_{u d} \lambda_{e \nu} \simeq 0.8024$, which seems too large as compared with the values derived in the previous sections.

\section{Conclusions}

In summary, we have studied several low-energy proccess to constraint the parameters of our version of the two-Higgs doublet model. Table 1 summarizes the bounds on the effective $H^{ \pm} f_{i} \bar{f}_{j}$ couplings defined in Eq.(3.1). To our knowledge this is the first study of generalized Yukawa couplings of the charged Higgs boson.

Through the use of these constraints, it has been possible to study several predictions of the model. In particular, we find that the model predicts $\mathrm{BR}(c \rightarrow$ 
$u+\gamma)<10^{-11}$, which is much larger than the SM predictions [27]. Unfortunately, this result can not be tested at the proposed tau-charm factory [29]. The prediction of large FCNC for the u-type quarks is clearly different from the SM picture.

Similarly, we find that the contribution from a charged Higgs boson to the second-class current decay of the tau give the bound $B R \leq 10^{-5}$, which is consistent with the present experimental bound. If the $\lambda_{i j}{ }^{6} s$ saturate the bound on their values, the $\mathrm{BR}$ would be above the SM prediction, and this could be testable at the proposed tau-charm factories [29].

Finally, the neutron lifetime would require a too large contribution from the charged Higgs, which seems inconsistent with the bounds obtained in section 3.

As we mentioned in the text, by studying the Yukawa couplings of the charged Higgs, it may be possible to test the models that attempt to explain the fermion mass spectrum of the SM. Even if some model can explain the spectrum, it would require further predictions in order to be accepted as a viable theory of masses. Our proposal is to search for a charged Higgs and study its Yukawa couplings, to see if it has the form predicted in those models that use an ansatz for the fermionic mass matrices [14], which give definite predictions for the Yukawa interacti ons of the Higgs bosons. ${ }^{\# 9}$.

$\underline{\text { Acknowledgements: }}$ We acknowledge the finantial support from CONACYT(ME$\mathrm{XICO})$.

\#9 However, at present such comparison is not possible without knowing the charged Higgs mass, which appears in our definition of $\lambda^{\prime} s$. 


\section{REFERENCES}

1. S. Weinberg, Phys. Rev. Lett. 19 (1967) 1264; A. Salam in Elementary Particle Theory, ed. N. Southolm (Almquist and Wiksell, Stockholm, 1969), p. 367; S.L. Glashow, Nucl. Phys. 22 (1961) 579.

2. F. Abe et al., CDF Collaboration, Phys. Rev. Lett. 73 (1994) 225.

3. G. Coignet, Proceed. of the XVI Lepton Photon Symposium, Eds. P.Drell and D. Rubin, Cornell Univ. August 10-15 (1993).

4. M. Kobayashi and T. Maskawa, Prog. Theor. Phys. 49 (1973) 652.

5. G. Branco and M. Rebelo, Phys. Lett. 160B (1985) 117.

6. S. Glashow, J. Iliopoulus and L. Maiani, Phys. Rev. D2 (1970) 1285.

7. Particle Data Book, Phys. Rev. D45 part II (1992).

8. G. Eilam et al., Phys. Rev. Lett. 67 (1991) 1979.

9. J.L. Diaz-Cruz et al., Phys. Rev. D41 (1990) 891; G.Eilam et al., Phys. Rev. D44 (1991) 1473.

10. J.L. Diaz-Cruz and G. Lopez Castro, Phys, Lett, B301 (1993) 405; M. Luke and M. Savage, Phys. Lett. B307 (1993) 387.

11. H. Georgi, Hadronic Jour. 1 (1978) 155.; H. Haber, G.L. Kane, T. Sterling, Nucl. Phys. B161 (1979) 493; S. Bertolini, Nucl. Phys. B272 (1986) 77; J.F. Gunion, H.E. Haber, Nucl. Phys. B272 (1986) 1 .

12. For complete references see: J. Gunion et al., "The Higgs Hunter Guide", Addison-Wesley.

13. M. Sher and Y. Yuan, Phys. Rev. D44 (1991) 1461.

14. For an elementary presentation see: D. Cline, Scientific Am, Sept. 1994. 
15. W-S. Hou, Preprint PSI-PR-91-34 (1991); T.P. Cheng and M. Sher, Phys. Rev. D35 (1987) 3448; A. Antaramian L.J. Hall and A Rasin, Phys. Rev. Lett. 69 (1992) 1871; L.J. Hall and S. Weinberg, Phys. Rev. D48 (1993) 979.

16. P. Krawczyk and S. Pokorski, Nucl. Phys. B364 (1991) 10.

17. A. Schwarz, in Ref. 3

18. W. Marciano and A. Sirlin, Phys. Rev. Lett. 61 (1988) 1815.

19. W. Marciano and A. Sirlin, Phys. Rev. Lett. 71 (1993) 3629; D.A. Bryman, Comments on Nuc. and Part. Phys. 21 (1993) 101.

20. R. Decker, M. Finkemaier, Karlsruhe preprint TTP-53-25 (1993).

21. I. Bigi, B. Blok, M.A. Shifman and A. Vainshtein, CERN-TH.7082/93; UND-HEP-93-BIG06; UMN-TH-1225/93; TPI-MINN-93/53-T; TechnionPh-93-40; October 1993.

22. B. Grinstein, N. Isgur, M.B. Wise, Phys. Rev. Lett. 56 (1986) 298; M. Wirbel B. Stech, M. Bauer, Z. Phys. C29 (1985)627; J.G. Körner and G.A. Schuler, Z. Phys. C46 (1990) 93.

23. V. Luth, in Ref. 3.

24. CLEO Collaboration, A. Bean et. al., Phys. Rev. Lett. 70 (1993) 2681.

25. ALEPH Collaboration, contributed paper XXVI Intern. Conf. on High Energy Physics (Dallas, TX, August 1992).

26. G. Isidori, Phys. Lett. B298 (1993) 409.

27. S. Egli, C. Grab, F. Ould-Saada, H. Simma, D. Wyler, Preprint ETHZIMPPR/92-1.

28. Wei-Shu Hou and R.S. Willey, Nucl. Phys. B326 (1989) 54; N. G. Deshpande and G. Eilam, Phys, Rev. D26 (1982) 2463. 
29. Workshop on the Tau-Charm-Factory, Marbella, Spain, June (1993).

30. M. Artuso et. al., CLEO Collaboration, Phys. Rev. Lett. 69 (1992) 3278.

31. Y. Meurice, Phys. Rev. D36 (1987) 2780; C.A. Domínguez, Phys. Rev. D20 (1979) 802; Y. Meurice, Mod. Phys. Lett. A2 (1987) 699; Phys. Rev. D36 (1987) 2780; A. Bramon, S. Narison and A. Pich, Phys. Lett. B196 (1987) 543; A. Pich, Phys. Lett. B196 (1987) 561; E. Berger and H. J. Lipkin, Phys. Lett. B189 (1987) 226; J.L. Diaz Cruz and G. López Castro, Modern Phys. Lett. A6 (1991) 1605.

32. Y. Meurice, Phys. Rev. D36 (1987) 2780.

33. See for example: D. Thompson, J. Phys. G: Nucl. Part. Phys. 16 (1990) 1423.

34. H. Haber, G. L. Kane, T. Sterling, Nucl. Phys, B161 (1979) 493.

35. D. Wilkinson, Nucl. Phys. A377 (1982) 474. 


\section{FIGURE CAPTIONS}

1) Feynman graphs for the contribution of charged Higgs to the 1-loop amplitude for $c \rightarrow u+\gamma$.

\section{TABLE CAPTIONS}

1: Summary of bound the $\lambda_{i j}$ 's obtained from low energy data as explained in the text. 
This figure "fig1-1.png" is available in "png" format from: http://arxiv.org/ps/hep-ph/9509229v1 